\title{
TRAP1 and the proteasome regulatory particle TBP7/Rpt3 interact in the endoplasmic reticulum and control cellular ubiquitination of specific mitochondrial proteins
}

\author{
MR Amoroso ${ }^{1}$, DS Matassa ${ }^{1}$, G Laudiero ${ }^{1}$, AV Egorova ${ }^{2}$, RS Polishchuk ${ }^{2}$, F Maddalena ${ }^{3}$, A Piscazzi ${ }^{4}$, S Paladino ${ }^{5,6}$, D Sarnataro ${ }^{5,6}$, \\ C Garbi ${ }^{5}, M_{\text {Landriscina }}{ }^{*, 4}$ and F Esposito ${ }^{*, 1}$
}

Tumor necrosis factor receptor-associated protein-1 (TRAP1) is a mitochondrial (MITO) antiapoptotic heat-shock protein. The information available on the TRAP1 pathway describes just a few well-characterized functions of this protein in mitochondria. However, our group's use of mass-spectrometric analysis identified TBP7, an AAA-ATPase of the 19S proteasomal subunit, as a putative TRAP1-interacting protein. Surprisingly, TRAP1 and TBP7 colocalize in the endoplasmic reticulum (ER), as demonstrated by biochemical and confocal/electron microscopic analyses, and interact directly, as confirmed by fluorescence resonance energy transfer analysis. This is the first demonstration of TRAP1's presence in this cellular compartment. TRAP1 silencing by short-hairpin RNAs, in cells exposed to thapsigargin-induced ER stress, correlates with upregulation of BiP/Grp78, thus suggesting a role of TRAP1 in the refolding of damaged proteins and in ER stress protection. Consistently, TRAP1 and/or TBP7 interference enhanced stress-induced cell death and increased intracellular protein ubiquitination. These experiments led us to hypothesize an involvement of TRAP1 in protein quality control for mistargeted/misfolded mitochondria-destined proteins, through interaction with the regulatory proteasome protein TBP7. Remarkably, expression of specific MITO proteins decreased upon TRAP1 interference as a consequence of increased ubiquitination. The proposed TRAP1 network has an impact in vivo, as it is conserved in human colorectal cancers, is controlled by ER-localized TRAP1 interacting with TBP7 and provides a novel model of the ER-mitochondria crosstalk.

Cell Death and Differentiation (2012) 19, 592-604; doi:10.1038/cdd.2011.128; published online 7 October 2011

Tumor necrosis factor receptor-associated protein-1 (TRAP1) was initially identified as a TNF-receptor-associated protein and is a member of the heat-shock protein-90 (HSP90) chaperone family. ${ }^{1,2}$ Through an mRNA-differential display analysis between oxidant-adapted and control osteosarcoma cells, our group identified, among other proteins, TRAP1, whose expression was highly induced upon oxidant adaptation. ${ }^{3}$ Furthermore, TRAP1 showed antioxidant and antiapoptotic functions, ${ }^{4}$ while an involvement of this mitochondrial (MITO) chaperone in the multi-drug resistance of human colorectal carcinoma (CRC) cells was also established. ${ }^{5}$

Little is known about TRAP1 signal transduction: the first most important finding on TRAP1 function came from studies by the Altieri's group, which identified TRAP1 as a member of a cytoprotective network selectively active in the mitochondria of tumor tissues. ${ }^{6}$ The same group has recently proposed TRAP1 as a novel molecular target in localized and metastatic prostate cancer, ${ }^{7}$ and is now involved in a promising preclinical characterization of mitochondria-targeted smallmolecule HSP90 inhibitors. ${ }^{8,9}$ Besides some well-characterized TRAP1 functions in mitochondria, during preparation of this manuscript it was reported that interference by HSP90 chaperones triggers an unfolded protein response (UPR) and activates autophagy in the mitochondria of tumor cells. ${ }^{10} \mathrm{~A}$ putative role of TRAP1 in endoplasmic reticulum (ER) stress control was concomitantly suggested by Takemoto et al., ${ }^{11}$ even though no evidence regarding the mechanisms involved was provided in this study.

A proteomic analysis of TRAP1 co-immunoprecipitation (co-IP) complexes was performed in our laboratory, in order to

\footnotetext{
${ }^{1}$ Department of Biochemistry and Medical Biotechnologies, University of Naples Federico II, Via Pansini 5, Naples 80131, Italy; ${ }^{2}$ Telethon Institute of Genetics and Medicine (TIGEM), Via Pietro Castellino 111, Naples 80131, Italy; ${ }^{3}$ IRCCS CROB, Rionero in Vulture, Italy; ${ }^{4}$ Clinical Oncology Unit, Department of Medical Sciences, University of Foggia, Foggia, Italy; ${ }^{5}$ Department of Biology and Molecular and Cellular Pathology, University of Naples Federico II, Naples, Italy and ${ }^{6} \mathrm{CEINGE}$ Biotecnologie Avanzate SCARL, Naples, Italy

${ }^{*}$ Corresponding authors: F Esposito, Dipartimento di Biochimica e Biotecnologie Mediche, Università di Napoli Federico II, Via Pansini 5, Napoli 80131, Italy. Tel: + 39081746 3145; Fax: + 39081746 4359; E-mail: franca.esposito @unina.it

or M Landriscina, Dipartimento di Scienze Mediche e del Lavoro, Università degli Studi di Foggia, Viale Pinto, 1, Foggia 71100, Italy. Tel: +39 0881 736241; Fax: + 390881 733614; E-mail: m.landriscina@ unifg.it

Keywords: TRAP1; TBP7; mitochondria/ER crosstalk; protein quality control; ubiquitination; apoptosis

Abbreviations: TRAP1, tumor necrosis factor receptor-associated protein-1; UPR, unfolded protein response; ER, endoplasmic reticulum; MS, mass spectrometry; FRET, fluorescence resonance energy transfer; EM, electron microscopy; CRC, colorectal carcinoma; TG, thapsigargin; Ub, ubiquitin; MTS, mitochondrial targeting sequence; UPS, ubiquitin-proteasome system; shRNA, short-hairpin RNA; GAPDH, glyceraldehyde-3-phosphate dehydrogenase; FITC, fluorescein isothiocyanate; MITO, mitochondrial; CYTO, cytosolic; S, supernatant; P, pellet; PM, post-mitochondrial

Received 20.4.11; revised 18.8.11; accepted 22.8.11; Edited by N Chandel; published online 07.10.11
} 
further characterize the TRAP1 network and evaluate protein interactors relevant for its roles. Among several other proteins, a novel MITO isoform of Sorcin, a calcium-binding protein, was identified as a new TRAP1 'ligand' and a cytoprotective function against apoptosis induced by antiblastic agents was recently demonstrated for this protein by our group. ${ }^{12}$ In the present paper, we characterize another new interaction of TRAP1 with TBP7/ATPase-4/Rpt3, an S6 ATPase protein of the proteasome regulatory subunit. ${ }^{13,14}$ TBP7 was first identified as a novel synphilin-1-interacting protein, ${ }^{13}$ so a functional role in Parkinson's disease was proposed for this protein. However, not many novel results became available subsequently on TBP7 function.

Altogether, (i) the absence of TBP7 in mitochondria; (ii) its still uncharacterized function as a regulatory protein; and (iii) its association with TRAP1 prompted us to analyze the subcellular localization of TRAP1/TBP7 interaction and to investigate its functional role. Several studies have described a function of HSP in the control of gene expression, ${ }^{15,16}$ and recent evidence demonstrated the importance of $19 \mathrm{~S}$ ATPases in the transcription machinery, as well as their additional regulatory mechanisms in mammalian transcription. ${ }^{17}$ Therefore, we hypothesized that the TRAP1/TBP7 interaction might have a role in protein quality control and cellular ubiquitination. Moreover, the finding that the two proteins directly interact in the ER further supports our hypothesis, as it is known that misfolding of proteins is tightly controlled by a large number of molecular chaperones and, if the quality control fails, they are ubiquitinated and degraded by the proteasome. ${ }^{18}$ This paper, for the first time, describes the presence of TRAP1 on the outer side of the ER and shows the functional role that TRAP1 has in the quality control of proteins destined to the mitochondria, and in the regulation of intracellular protein ubiquitination, through interaction with TBP7.

\section{Results}

TRAP1 and TBP7 colocalize and directly interact in the ER. The 'fishing for partners' strategy combined with mass spectrometric procedures performed to identify TRAP1

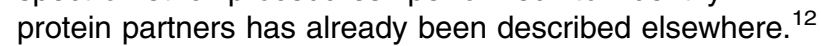

Among the putative TRAP1-binding partners, we focused our attention on a protein of about $50 \mathrm{kDa}$ identified by our LC-MS/MS analysis as S6/TBP7/ATPase-4/Rpt3. ${ }^{19}$ To confirm that this proteasome subunit was indeed a TRAP1interacting protein, we performed western blot (WB) and co-IP analyses in total extracts from HCT116 colon carcinoma cells (Figure 1A). As TRAP1 is localized in mitochondria, subfractionation of HCT116 cellular extracts was performed. Surprisingly, WB analysis of sub-cellular compartments failed to demonstrate the presence of the regulatory protein in mitochondria, but identified TBP7 both in the cytosol and microsomal fraction (Figure 1B). Co-IP analyses from ER fraction confirmed the TRAP1/TBP7 interaction in the ER (Figure 1C).

The TRAP1/TBP7 molecular interaction was further investigated by using a fluorescence resonance energy transfer (FRET) approach in fixed cells (Figure 1D). ${ }^{20}$ In cells coexpressing wild-type TRAP1 and TBP7 we found about $12 \%$ of FRET efficiency (Figure 1D) indicating that TRAP1 and TBP7 are close enough to allow energy transfer. Interestingly we found FRET exclusively when we bleached ER regions, which we selected on the basis of their morphological features (one example in Figure 1D, a-f). Thus, these data indicate that TRAP1 and TBP7 directly interact with each other and this interaction occurs specifically in the ER compartments. Furthermore, according to the biochemical data described below in the paper (Supplementary Figure 1 and Figure $4 \mathrm{H}$ ), we found no FRET between TRAP1 and a mutant form of TBP7 (Figure 1D).

As it had never been reported previously, we aimed to further confirm the novel localization of this MITO chaperone in the ER through electron microscopy (EM) and confocal microscopy analyses (Figures $1 \mathrm{E}$ and $\mathrm{F}$ ). To further evaluate whether TRAP1 is associated with the ER membranes, cells stably expressing TRAP-HA vectors were prepared for immuno-EM. Labeling with an anti-HA antibody revealed significant amounts of TRAP1 in mitochondria (Figure 1E, a and $b$, arrowheads), as has been reported previously. ${ }^{6}$ In addition, we found that TRAP1 was distributed throughout the elongated membrane profiles (Figure 1E, a, arrows) that on the basis of their ultrastructural features (such as attached ribosomes) can be attributed to the rough ER compartment. Moreover, gold particles - indicating TRAP1 molecules - were detected along the nuclear envelope (Figure 1E, b, arrows), considered to be part of the ER membrane network. A careful examination of the density of immuno-gold labeling (in arbitrary units; average \pm S.D.) over different intracellular membranes allowed us to demonstrate that indeed TRAP1 was enriched in mitochondria $(2.53 \pm 0.34)$ as it has already been reported. However, significant labeling density was also calculated for ER membranes $(1.02 \pm 0.22)$ as shown in Figure 1E. Taken together, the EM observations are in line with the above results showing association of TRAP1 with the ER (Figures $1 \mathrm{~A}-\mathrm{C}$ ). Accordingly, immunofluorescence confocal microscopy analysis showed colocalization of TBP7 with TRAP1 and with the ER protein calnexin, thus confirming ER localization further (Figure 1F).

Altogether, these findings demonstrate that the interaction between TRAP1 and TBP7 occurs in the ER, but are unable to reveal a detailed localization in this context. To further evaluate the 'topology' of TRAP1 and TBP7 in the ER, a biochemical assay based on protease digestion was performed. Figure $1 \mathrm{G}$ shows that both proteins are sensitive to a proteinase-K digestion, whereas calnexin, a well-known ERresident protein, is undigested. These approaches allowed us to demonstrate that TRAP1 and TBP7 are located on the outside of the ER. Moreover, alkaline treatment of ER fractions to remove peripheral membrane proteins allowed us to demonstrate that both TRAP1 and TBP7 are loosely associated to ER membranes (Figure $1 \mathrm{H}$ ).

TRAP1 interference sensitizes CRC cells to TG-induced ER stress. We therefore investigated the functional role of the TRAP1/TBP7 interaction. Several data suggest that ER stress, worsened by the high ROS concentrations in this sub-cellular compartment, is controlled by networks of molecular chaperones. ${ }^{21}$ Considering the previously described protective role of TRAP1 against several 

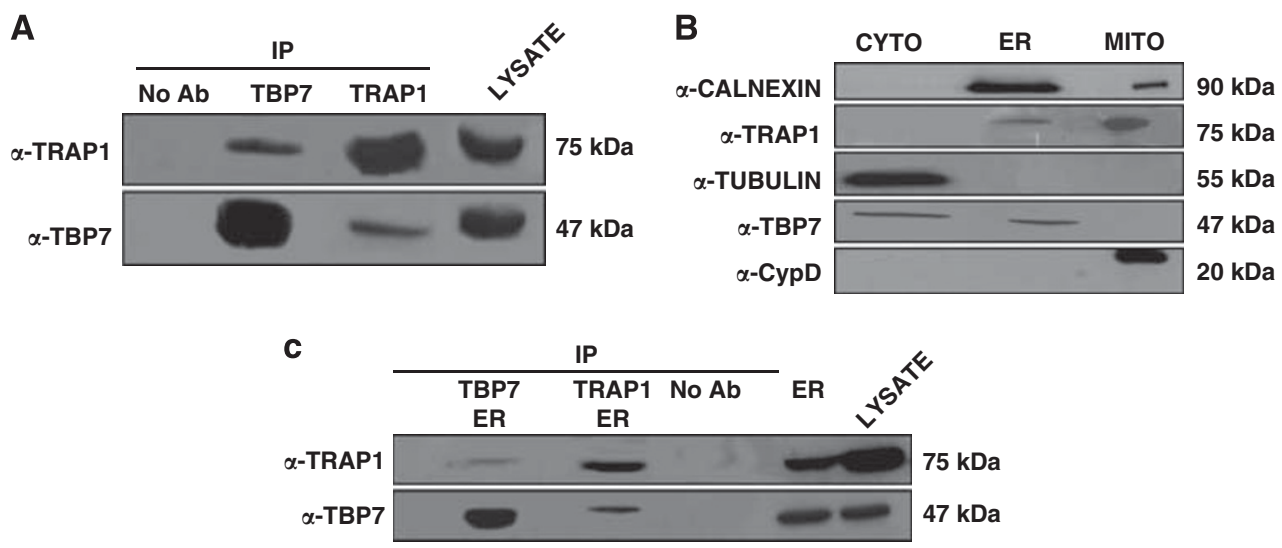

D

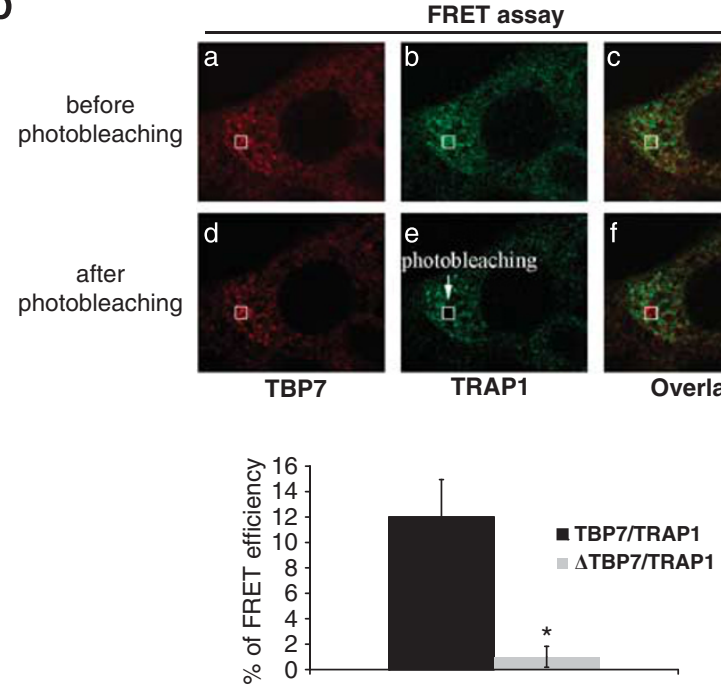

F

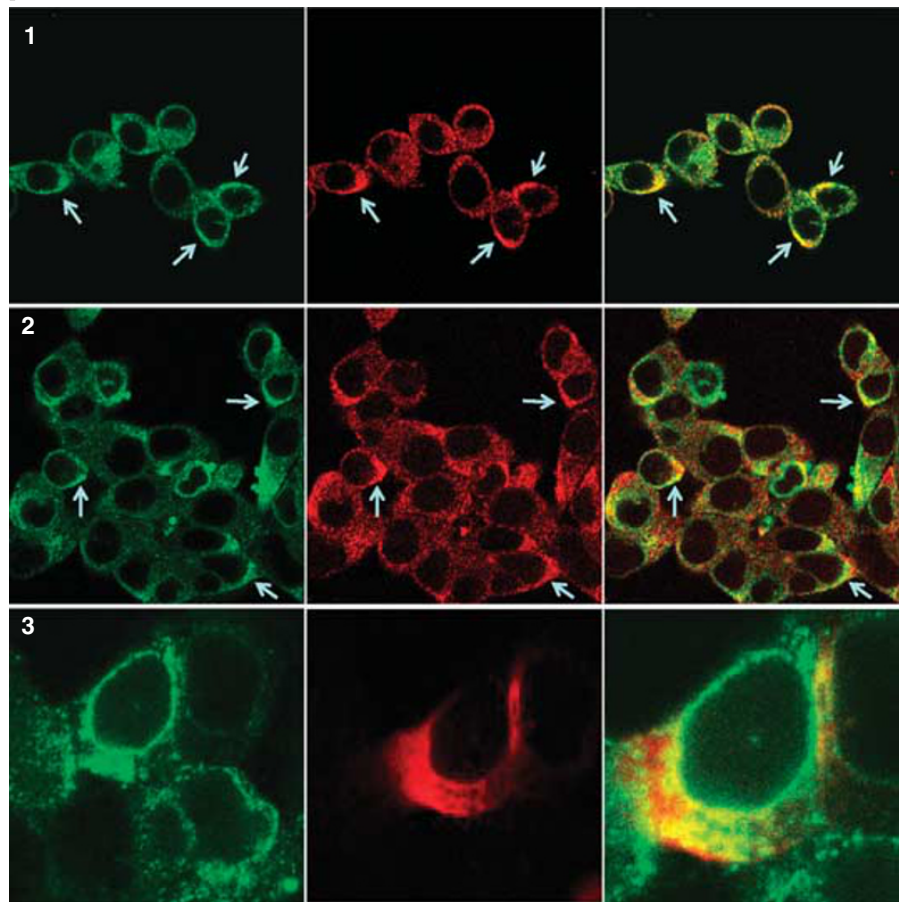

E
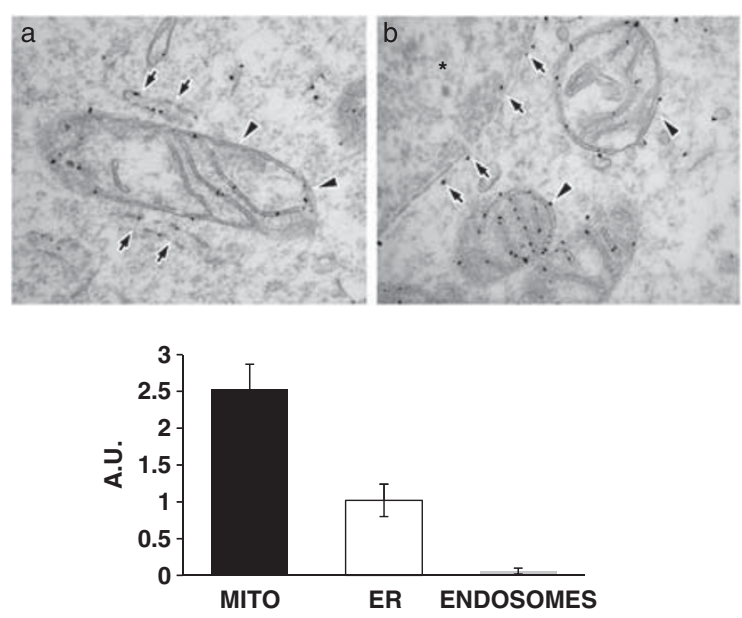

G

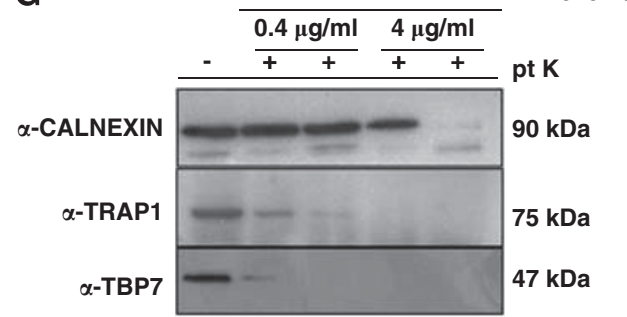

H

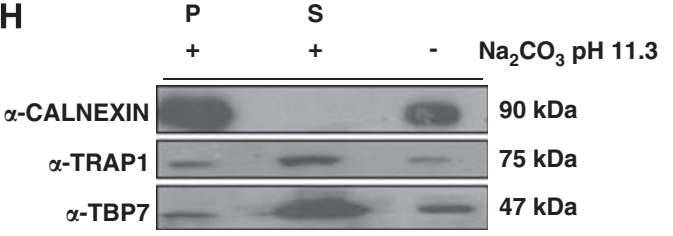


stresses, including oxidative stress through its antioxidant functions, ${ }^{4,22}$ we hypothesized that the TRAP1/TBP7 interaction constituted an additional control to check the state/folding of proteins damaged in the ER. In order to induce protein misfolding in the ER, we induced ER stress by using thapsigargin (TG), a well-known agent that mobilizes $\mathrm{Ca}^{2+}$ from the ER. ${ }^{23}$ As a marker of ER stress we analyzed the expression of BiP/Grp78, a major ER chaperone protein essential for protein quality control in the ER, as well as a central regulator of the UPR. ${ }^{24}$ Figure 2 shows that $\mathrm{BiP}$ mRNA expression is strongly increased, upon TG treatment, in TRAP1-interfered cells as compared with the respective control (Figures $2 a$ and $b$ ). Interestingly, a rescue of this phenotype is achieved upon TRAP1 re-addition (Figure 2b). These results demonstrate that TRAP1 knockdown sensitizes cells to TG-induced ER stress, thus suggesting an involvement of this protein in ER stress response, as reported recently. ${ }^{10,11}$

We and other groups have previously demonstrated the protective roles of TRAP1 against cell death induced by several agents. ${ }^{4,6}$ Therefore, we addressed whether TRAP1interfered cells become more sensitive to apoptosis, as a possible and expected consequence of increased ER stress, and whether TBP7 might have a role in this process. Table 1 shows that TRAP1 and TBP7 interference sensitized HCT116 cells to TG-induced apoptosis. Noteworthy, a similar result was observed upon cell treatment with oxaliplatin, a genotoxic agent (Table 1). Taken together, all these observations confirm the well-known role of TRAP1 in the protection against stress-induced cell death and highlight a new role for regulatory proteasome proteins in apoptotic control.

TRAP1/TBP7 control of intracellular protein ubiquitination. We then hypothesized that, if the level of protein damage upon ER stress is too severe to be counteracted/ repaired by TRAP1 refolding, increased protein degradation might occur, and increased ubiquitination levels of intracellular proteins could be observed. Indeed, tight regulation of protein ubiquitination by TRAP1/TBP7 does occur: in fact, a low-ubiquitin (Ub) signal is observed in
TRAP1-containing cells, whereas TRAP1 interference strongly increases general protein ubiquitination levels (Figure 3).

The control of ubiquitination of intracellular proteins by TRAP1/TBP7 is a general phenomenon as it is present in total
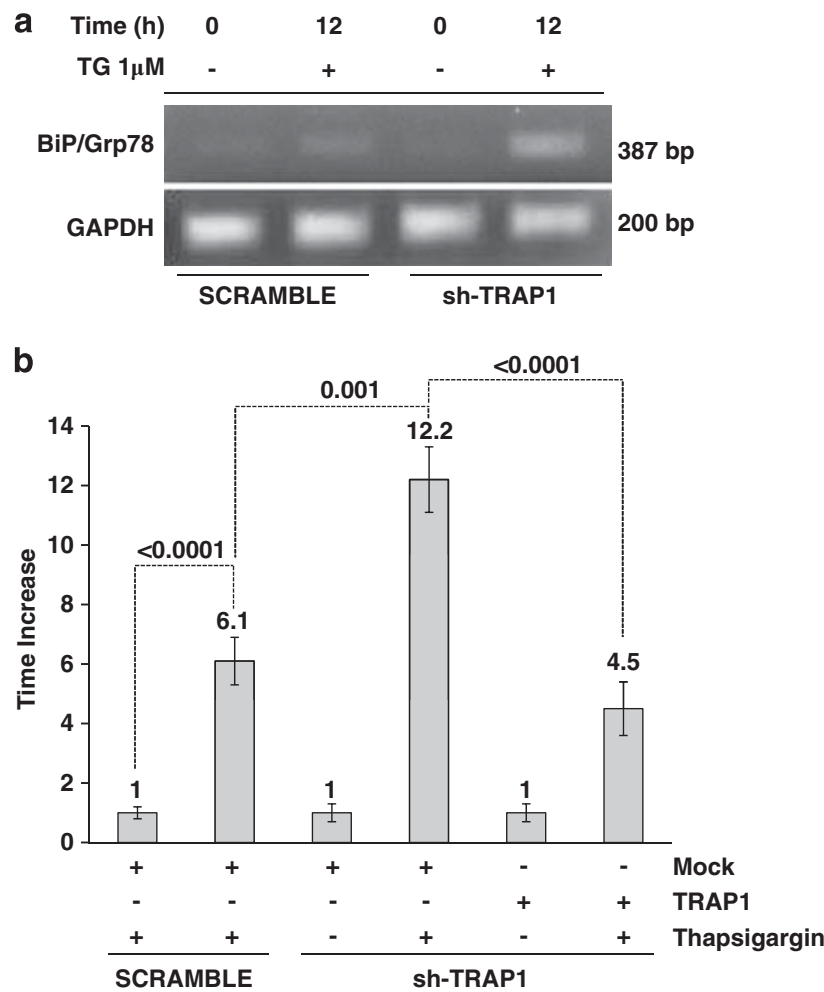

Figure 2 BiP mRNA levels in sh-TRAP1 stable clones. (a) Semi-quantitative RT-PCR analysis of BiP/Grp78 mRNA expression in sh-TRAP1 stable clones with respect to scrambled transfectants after 12-h treatment with $1 \mu \mathrm{M} \mathrm{TG}$. As control, the levels of GAPDH transcript were analyzed. (b) Real-time RT-PCR analysis of BiP/Grp78 mRNA expression in scrambled and sh-TRAP1 HCT116 cells exposed to $1 \mu \mathrm{M}$ TG for $12 \mathrm{~h}$ and in sh-TRAP1 HCT116 cells transfected with TRAP1 cDNA before treatment with TG. The $P$-values indicate the statistical significance between different BiP/Grp78 levels under the indicated conditions

Figure 1 TRAP1 and TBP7 interact and colocalize in the ER. (A) Total HCT116 lysates were harvested and immunoprecipitated using anti-TRAP1 and anti-TBP7 antibodies as described under Materials and Methods, separated by SDS-PAGE and immunoblotted using the indicated mouse monoclonal anti-TRAP1 and mouse monoclonal anti-TBP7 antibodies. No Ab, total cellular extracts incubated with A/G plus agarose beads without antibody; IP, immunoprecipitation using the corresponding antibodies. (B) Total HCT116 lysates were fractionated into MITO, CYTO and microsomal (ER) fractions as described under Materials and Methods, separated by SDS-PAGE and immunoblotted using mouse monoclonal anti-TRAP1 and mouse monoclonal anti-TBP7 antibodies. The purity of the fractions was assessed by using mouse monoclonal anti-tubulin, goat polyclonal anti-CypD, rabbit polyclonal anti-calnexin antibodies specific for the single subcellular compartments. (C) TRAP1 and TBP7 co-IP analysis on the microsomal fraction (ER), obtained as described under Materials and Methods. WB of immunoprecipitates was performed by using the indicated antibodies. (D) TRAP1/TBP7 direct interaction. FRET was measured by using the acceptor photo-bleaching technique as described under Materials and Methods. The images show the signal of TBP7 (red) and TRAP1 (green) before (a-c) and after photo-bleaching (d-f). The selected ROI for bleaching was indicated. Energy transfer efficiency was measured in cells transiently co-transfected with TRAP1 and either TBP7 or its mutant form ( $\triangle \mathrm{TBP7}$-Flag), and is expressed in \% as mean of three independent experiments. Error bars: \pm S.D.; ${ }^{*} P<0.0001$. (E) ER Distribution of TRAP1 in HCT116 cells (EM). Cells expressing TRAP-HA vector were fixed and prepared for immuno-EM (see Materials and Methods). Labeling with the anti-HA antibody revealed significant amount of TRAP1 in mitochondria (a, b, arrowheads). In addition, TRAP1 was distributed throughout the elongated membrane profiles ( $\mathrm{a}$, arrows) that on the basis of their ultrastructural features (such as attached ribosomes) can be attributed to the rough ER compartment, and detected along the nuclear envelope (b, arrows). The density of immuno-gold labeling (in arbitrary units; average \pm S.D.) in mitochondria (MITO), ER and endosomes (as a negative control) is reported in the lower histogram. (F) ER TRAP1/TBP7 colocalization (confocal microscopy). Immunofluorescence shows colocalization of TBP7 with TRAP1 and with the ER protein calnexin. In Panel-1, a double immunofluorescent staining is shown for TRAP1 (green) and TBP7 (red). In Panel-2, a double immunofluorescent staining is shown for calnexin (green) and TBP7 (red). In cells expressing the Myc-tagged TRAP1 construct (red) the protein co-distributes to a great extent with endogenous calnexin (green, Panel-3). (G and $\mathbf{H}$ ) Biochemical characterization of TRAP1/TBP7 'topology' in the ER. WB of HCT116 microsomal fractions treated with $0.4 \mu \mathrm{g} / \mathrm{ml}$ or $4 \mu \mathrm{g} / \mathrm{ml}$ proteinase-K (pt K) $\pm 1 \% \mathrm{NP}-40$ for $20 \mathrm{~min}$ on ice $(\mathbf{G})$ or with $100 \mathrm{mM} \mathrm{Na}_{2} \mathrm{CO}_{3}(\mathrm{pH} 11.3)$ for $30 \mathrm{~min}(\mathbf{H})$ as described under Materials and Methods. Specific proteins were revealed using the indicated antibodies. $(\mathbf{H}): \mathrm{S}$, supernatant; $\mathrm{P}$, pellet 
Table 1 Rates of apoptotic cell death in CRC HCT116 cells treated with $1 \mu \mathrm{M}$ TG for $24 \mathrm{~h}$ or with $10 \mu \mathrm{M}$ oxaliplatin (I-OHP) for $48 \mathrm{~h}$ upon transient (siRNA) or stable (shRNA) downregulation of TRAP1 or TBP7

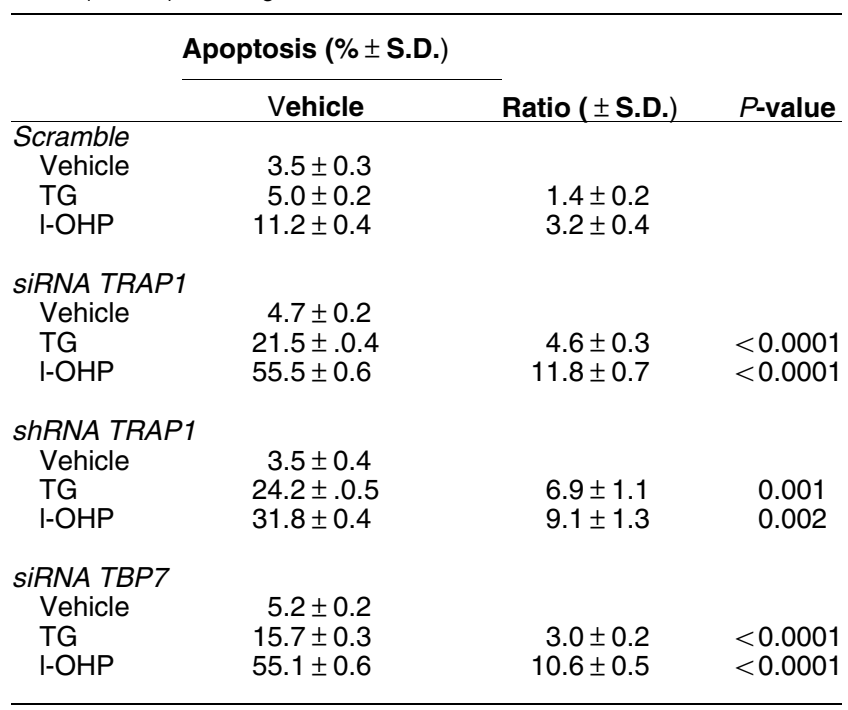

Ratios are calculated between rates of apoptosis in drug- and vehicle-treated cells. The $P$-values indicate the statistical significance between the ratios of apoptosis in siRNA-transfected cells and the respective scrambled controls.

extracts (Figure 3a). Interestingly, both TRAP1 and TBP7 seem to have a critical role in the regulation of protein ubiquitination. In fact, upon transfection of TRAP1 expression vectors in sh-TRAP1 stable transfectants a rescue of the highUb 'phenotype' is observed (Figure 3a). Sub-cellular fractionation allowed us to demonstrate that this regulatory role is more evident in the post-mitochondrial (PM) fraction (cytosol + microsomes), where TRAP1/TBP7 control is particularly necessary given the abundance of proteins translated, but often damaged. Conversely, the same regulation is not observed in MITO extracts even after re-transfection of TRAP1, likely because TBP7 is absent in these organelles (Figure $3 b$ ). These results confirm that TRAP1 function in the regulation of protein ubiquitination requires the presence of TBP7. Additionally, increased cellular levels of ubiquitinated proteins, very similar to those obtained in cells transiently transfected with TRAP1 siRNAs, were observed upon TBP7 SiRNA transfection (Figure $3 \mathrm{c}$ ), thus confirming the role of TBP7 in this regulation. Altogether, these results demonstrate that the TRAP1/TBP7 interaction is a useful and important checkpoint in which these two proteins concomitantly work to judge whether a protein can be repaired and reach the final destination or, if the damage is too severe, it needs to be degraded. Furthermore, despite the block of proteasome activity by MG132 treatment, the experiments shown in Figure 3 demonstrate that the regulation of protein ubiquitination by TRAP1 is not due to inhibition of proteasome function, as it is observed also in the absence of the inhibitory drug (Figure 3a). Indeed, this finding was confirmed by assaying the proteasome's activity in vitro using fluorescent substrates and extracts from scrambled- and TRAP1- or TBP7-interfered cells. The results shown in Figure $3 \mathrm{~d}$ demonstrate that neither TRAP1 nor TBP7 interference inhibits the proteasome's function.
More importantly we asked whether the control of protein ubiquitination by TRAP1/TBP7 requires TRAP1 MITO localization. To address this issue we generated the $\Delta 1-59$ deletion mutant of TRAP1 (in which the first 59 aa containing the MITO targeting sequences $\left(\mathrm{MTS}^{2}\right)$ were removed from the $\mathrm{N}$ terminus) yielding a TRAP1 mutant defective for MITO import, but still able to bind to TBP7 (Supplementary Figure 1 and Figures $4 a$ and $b$ ). Interestingly, transfection of this mutant in sh-TRAP1 stable clones rescued the heavy Ub levels present in TRAP1-interfered cells (Figure 4c). Conversely, another TRAP1 deletion mutant $(\Delta 101-221)$ was generated that keeps the MTS (Supplementary Figure 1) and, thus, is able to localize into mitochondria, but is unable to bind to TBP7, which, as shown previously, is absent in the MITO fraction. Transfection of the $\Delta 101-221$ mutant in sh-TRAP1 cells showed no changes in protein ubiquitination levels (Figures $4 d-f)$. These experiments provide proof of concept that the relevant amount of TRAP1 present in the ER fractions (see the EM qualitative/quantitative analyses shown in Figure 1e) is indeed involved in the regulation of protein ubiquitination through its binding to TBP7, whereas MITO TRAP1 does not influence Ub levels, according to the phenotype generated by the $\Delta 101-221$ TRAP1 deletion mutant. Finally, we demonstrated that transfection of the TRAP1 $\Delta 1-59$ mutant decreased the BiP/Grp78 mRNA levels present in sh-TRAP1 clones upon TG-induced ER stress. Figure $4 \mathrm{~g}$ shows that a rescue of $\mathrm{BiP} / \mathrm{Grp78} \mathrm{mRNA}$ levels is obtained upon transfection of the $\Delta 1-59$ mutant, whereas the $\Delta 101-221$ TRAP1 mutant with MITO localization not only is unable to counteract ER stress, but even further increased BiP/Grp78 levels.

Strikingly, a TBP7 deletion mutant (Supplementary Figure 1), unable to bind to TRAP1 (Figure 4h), yields, upon transfection in HCT116 cells (scrambled), a strong Ub pattern undistinguishable from the phenotype of sh-TRAP1 stable transfectants (Figure 4i), likely acting as dominant negative over the endogenous TBP7 protein's function.

Quality control of specific mitochondria-destined proteins by TRAP1/TBP7. Once we demonstrated the contribution of TRAP1/TBP7 to the regulation of ER stress/ apoptosis, with the consequent modulation of intracellular protein ubiquitination, we hypothesized that TRAP1 could be involved in a general control of protein stability. To this aim, pulse-chase experiments were performed in scrambled and sh-TRAP1 stable transfectants. However, a comparable pattern of degraded proteins is still observed $72 \mathrm{~h}$ after Met/Cys chase (Figure 5a). This unchanged overall protein stability led us to hypothesize that TRAP1 could be involved in the control of protein folding/stability for selective proteins, likely those directed to mitochondria. To evaluate this hypothesis, the levels of F1ATPase, a nuclear-encoded MITO protein and a potential TRAP1 interactor as suggested by MS analysis, ${ }^{12}$ were analyzed in cells in which TRAP1 expression was lowered by short-hairpin RNA (shRNA) interference. Interestingly, protein levels decreased in TRAP1 stably interfered cells (Figure $5 \mathrm{~b}$ and Supplementary Figure 2), whereas a rescue was obtained upon re-addition of the $\Delta 1-59$ TRAP1 mutant but not by the 1101-221 TRAP1 mutant (Supplementary Figure 3). These findings confirm that the regulation of the observed 


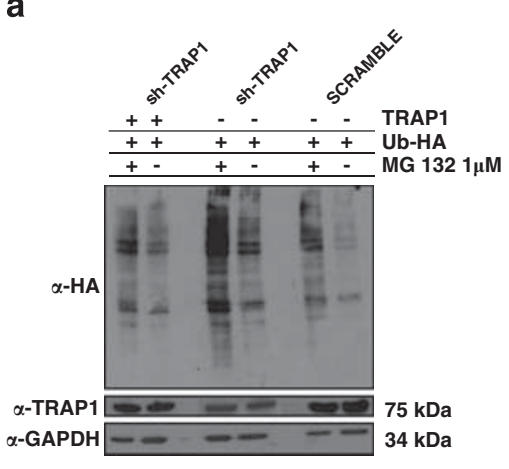

C

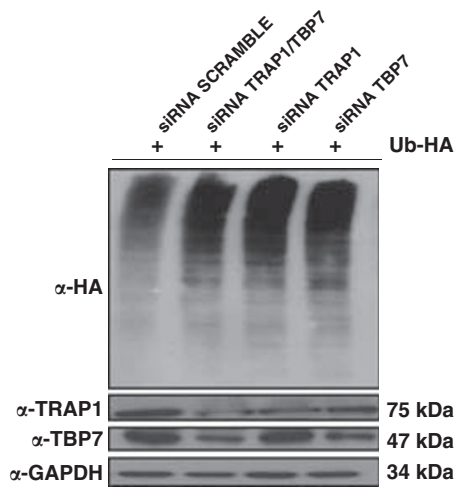

b
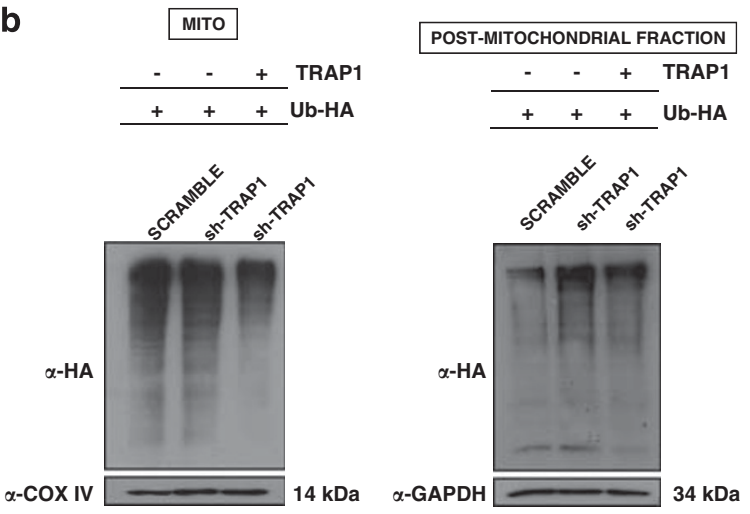

d

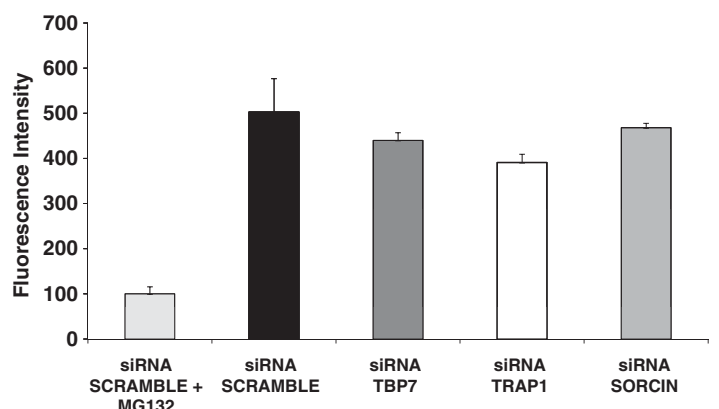

Figure $3 \mathrm{Ub}$ levels in HCT116 cells. (a) Total cell lysates from sh-TRAP1 and scrambled HCT116 stable clones were transfected with either an HA-tagged Ub vector $(\mathrm{Ub}-\mathrm{HA})$ or with TRAP1 expression vectors; treated with $1 \mu \mathrm{M}$ MG132 for $24 \mathrm{~h}$; harvested $48 \mathrm{~h}$ after transfection; and subjected to immunoblot using rabbit polyclonal anti-HA antibodies. The same filter was re-probed using mouse monoclonal anti-GAPDH antibodies for normalization of cell lysates. Three independent experiments were performed, with similar results. (b) Sub-cellular fractionation was obtained from sh-TRAP1 and scrambled HCT116 stable transfectants treated as described in panel a. The extracts from the PM fraction (microsomes + CYTO fraction) and mitochondria (MITO, see Materials and Methods) were separated by SDS-PAGE and immunoblotted using a rabbit polyclonal anti-HA antibody to detect $\mathrm{Ub}$ levels. The purity of fractions was verified by using mouse monoclonal anti-COX IV or mouse monoclonal anti-GAPDH antibodies. Three independent experiments were performed, with similar results. (c) HCT116 cells were co-transfected with a Ub-HA vector and an siRNA negative control (scramble), or with siRNAs specific for TRAP1, TBP7, or both (as indicated) and total cell lysates were harvested after $48 \mathrm{~h}$ from transfection. Total lysates were subjected to SDS-PAGE and immunoblotted using rabbit polyclonal anti-HA antibodies to detect total Ub levels. The same filter was re-probed using mouse monoclonal anti-GAPDH antibodies for normalization of cell lysates, and using mouse monoclonal anti-TRAP1 and mouse monoclonal anti-TBP7 antibodies. (d) Proteasome activity is not affected by TRAP1 and TBP7 silencing. Total cellular extracts were prepared after $48 \mathrm{~h}$ of transfection with specific siRNA for TRAP1, TBP7 or Sorcin, as control, or with an siRNA negative control (scramble), and incubated in the presence of assay buffer and the fluorogenic substrate Suc-LLVY-AMC, as described under Materials and Methods. Samples were analyzed in triplicate using an excitation wavelength of $360 \mathrm{~nm}$ and an emission wavelength of $450 \mathrm{~nm}$ to detect chymotryptic proteasome activity. The data represent the mean of three independent experiments

phenomena occurs in the cytosolic (CYTO) compartment and support our model. Accordingly, the protein levels of p18 Sorcin, another MITO protein, recently identified by our group as a novel MITO Sorcin isoform interacting with TRAP1, ${ }^{12}$ decreased upon TRAP1 interference (Figure 5b, arrow). Of note, under the same experimental conditions, no differences were observed in the protein levels of the higher mobility p22 Sorcin isoform, which shares high homology with $\mathrm{p} 18$ Sorcin, but is not a MITO protein, neither is a TRAP1 'partner' (Figure 5b). ${ }^{12}$ Therefore, we hypothesize that the decreased expressions of F1ATPase and p18 Sorcin in the mitochondria of TRAP1-interferred cells were dependent on increased ubiquitination. To this aim, the respective ubiquitination levels in scrambled- and TRAP1-interfered cells were analyzed. Figures $5 \mathrm{c}$ and $\mathrm{d}$ show that both proteins are more ubiquitinated in sh-TRAP1 transfectants. Accordingly, increased levels of ubiquitinated F1ATPase were induced upon TBP7 interference (Figure 5e).

Finally, we evaluated whether the TRAP1/TBP7 interaction and the relative effects on protein levels would have an impact in vivo. To this aim, we analyzed our tissue collection of CRCs, previously characterized for TRAP1 and Sorcin expression by immunoblot analysis. ${ }^{12}$ We observed that all CRCs overexpressing the 18-kDa Sorcin isoform (11/59 cases) shared the concomitant upregulation of TRAP1. Therefore, we analyzed for TRAP1, Sorcin, F1ATPase and TBP7 expression the 11 Sorcin-overexpressing tumors and, as controls, other $14 \mathrm{CRC}$ specimens. Figure 6 reports the immunoblot analysis of the four proteins in four tumor samples representative of our tumor collection, whereas the expression profile of the four genes in $25 \mathrm{CRCs}$ is reported in Supplementary Table 1. Remarkably, the majority of the p18 Sorcin- and TRAP1-positive tumors showed upregulation of TBP7 (9/11 cases) and F1ATPase (8/11 cases). By contrast, among eight tumors with low expression of TRAP1, all showed low levels of TBP7 and p18 Sorcin and 7/8 showed low expression of F1ATPase. $\chi^{2}$-Test demonstrated a positive statistical correlation between the expression levels of TRAP1 and those of Sorcin, F1ATPase and TBP7 (Supplementary Table 1). 
Discussion

TRAP1 was identified by our group as one of the proteins involved in and important for the homeostasis of

a

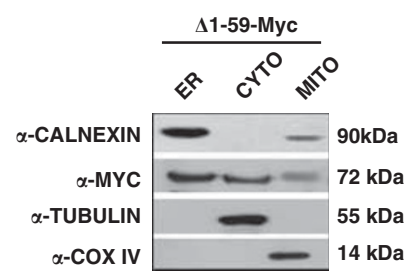

b

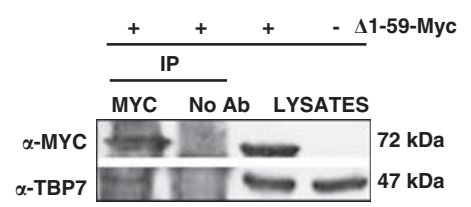

osteosarcoma cells adapted to mild oxidative stress. ${ }^{4}$ The control of the protein folding environment in sub-cellular organelles, such as mitochondria, is important for adaptive homeostasis and may participate in human diseases, but the

C

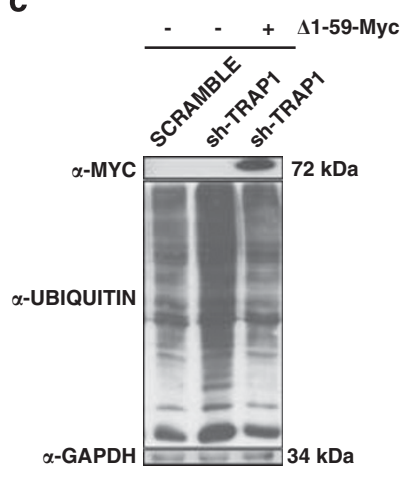

d

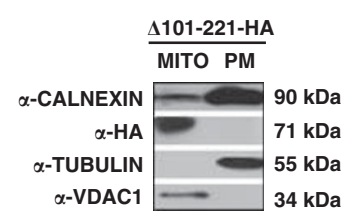

e

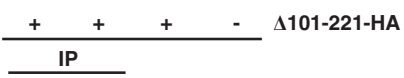

HA No Ab LYSATES

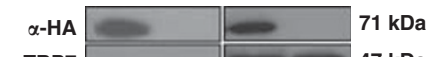

$\alpha$-TBP7

$47 \mathrm{kDa}$

f
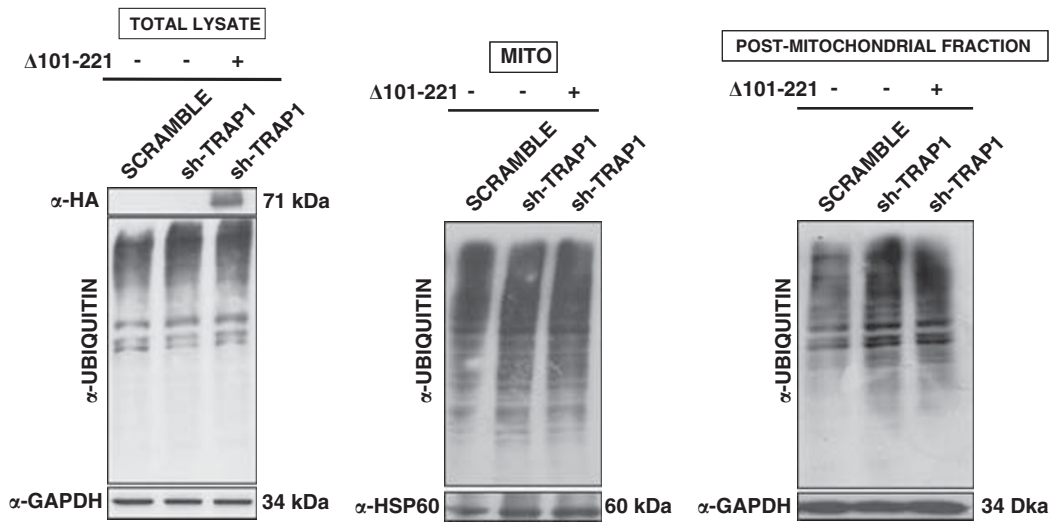

g

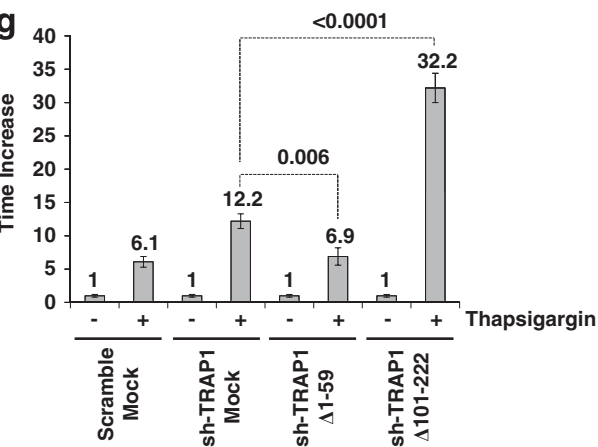

h

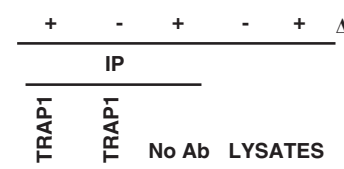
$\Delta$ TBP7-Flag

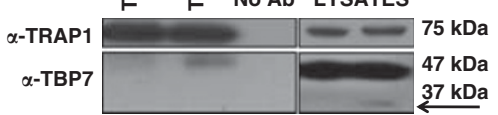


regulators of this process are still largely elusive. During the preparation of this manuscript, Altieri et al. ${ }^{10}$ demonstrated that selective targeting of HSP90 chaperones in mitochondria of human tumor cells triggered compensatory autophagy, and an organelle UPR enhanced tumor cell apoptosis initiated by death receptor ligation, and inhibited tumor growth in mice without detectable toxicity. These results reveal a novel role of HSP90 chaperones in the regulation of the protein folding environment in mitochondria of tumor cells.

Starting from the above observations and in agreement with Altieri's results, here we demonstrate an additional role of TRAP1 in protein quality control, acting on the outside of the ER. This TRAP1 function requires TBP7, a protein of the 19S proteasome regulatory subunit. As demonstrated by mass spectra analysis, this AAA-ATPase is a novel TRAP1interacting protein whose role is determinant in the quality control of proteins. In fact, the interference of either TRAP1 or TBP7 proteins resulted both in the induction of apoptosis in response to both TG-induced ER stress or oxaliplatin treatment, and in increased intracellular protein ubiquitination, which was selectively rescued by re-addition of TRAP1. Interestingly, TRAP1/TBP7 regulation of cellular ubiquitination is independent of modulation of proteasome function, as an in vitro assay of proteasome activity shows that TRAP1/ TBP7 interference does not affect proteasome functionality. The regulatory networks that control the protein folding machinery in mitochondria are still largely elusive. In this regard, the demonstration that regulation of protein quality control by TRAP1 is directed toward MITO proteins, expands the currently rather limited list of MITO proteasome targets. It is reasonable to hypothesize that multiple chaperone networks control the misfolding of proteins addressed to different sub-cellular compartments. In this study, an additional 'pre'-screening for proteins directed to mitochondria is characterized: if the protein is highly damaged and not successfully refolded by the chaperone machineries inside or outside the ER, including TRAP1, it will be identified by the regulatory proteasome protein, and targeted for degradation. TRAP1-containing supra-molecular complexes might be present just outside the mitochondria, and in the cellular compartment of the tight ER-mitochondria interface (Hayashi and $\mathrm{Su}^{25}$ and Figure 1e), where proteasomes have also been identified $^{26}$ and ensure that among all the proteins translated in the ER, only undamaged proteins could enter the mitochondria. Very recently, Takemoto et al. ${ }^{11}$ suggested that the MITO chaperone TRAP1 regulates the UPR in the $E R$, even though its presence in the ER has not been reported. Therefore, the demonstration that TRAP1 is present also on the external side of the ER is an important achievement strongly supporting its role in protection against stress.

A still unsolved question is the mechanistic link between TRAP1-dependent MITO adaptive response to stress and regulation of gene expression. Interestingly, some analyses have demonstrated the importance of 19S ATPases in the assembly of the transcription machinery. ${ }^{17}$ Alternatively, it could be hypothesized that MITO chaperones may regulate gene expression by modulating ER stress. In support of this hypothesis, our results suggest that TRAP1 may be involved in the ER stress response. Furthermore, an interaction between TRAP1 and Sorcin, a calcium-binding protein, was characterized by our group, ${ }^{12}$ which might contribute to a regulatory role of the TRAP1 and TRAP1-interacting proteins in ER stress induced by perturbation of calcium homeostasis (Maddalena et al., unpublished data) and protein quality control.

We did not observe any strong differences in MITO protein ubiquitination upon modulation of TRAP1 levels. Quality control of MITO proteins must be monitored by molecular chaperones. ${ }^{27}$ Only very small numbers of MITO proteins are currently known to be degraded in a proteasome-dependent manner, ${ }^{28}$ so a more focused effort to identify such additional substrates might dramatically expand the list of MITO proteasome targets. Our results demonstrate that expression of p18 Sorcin and F1-ATPase is decreased upon TRAP1 interference as a consequence of their increased ubiquitination. The identification of TRAP1/TBP7-specific 'substrates' strongly contributes to the complex study of MITO protein quality control. One reason for ubiquitination of MITO proteins may be that when mitochondria-destined proteins are

Figure 4 The TRAP1/TBP7 interaction in the ER is required for control of protein ubiquitination and ER stress. (a and d) Sub-cellular localization of $\Delta 1-59-M y c / \Delta 101-221-H A$ mutants. HCT116 cells were transfected with the $\Delta 1-59$-Myc (a) or $\Delta 101-221$-HA (d) TRAP1 mutants; sub-fractionated into MITO, CYTO and microsomal (ER) fractions (a), or MITO and PM (cytosol + microsomes) fractions (d), as described under Materials and Methods; separated by SDS-PAGE; and immunoblotted using the indicated antibodies to verify the expression of mutants and the purity of fractions. For details on procedures for generation of the mutants see Materials and Methods. (b, e) Interaction between $\Delta 1-59-M y c / \Delta 101-$ 221-HA mutants and TBP7. HCT116 cells were transfected with $\triangle 1-59-$ Myc (b) or $\triangle 101-221-H A(e)$ TRAP1 mutants, harvested and immunoprecipitated using anti-Myc or anti-HA antibodies as described under Materials and Methods. Immunoprecipitates were separated by SDS-PAGE and immunoblotted using the indicated antibodies. No Ab, total cellular extracts incubated with A/G plus agarose beads without antibody; IP, immunoprecipitation with the corresponding antibodies. Three independent experiments were performed, with similar results. (c) Ubiquitination levels upon transfection of the $\triangle 1-59$-Myc TRAP1 deletion mutant. Total lysates from HCT116 scrambled, sh-TRAP1 stable clones and sh-TRAP1 cells transfected with the $\Delta 1-59-M y c$ TRAP1 mutant were subjected to immunoblot analysis using mouse monoclonal anti-Ub antibodies to detect total ubiquitination levels and with an anti-GAPDH antibody for normalization of cell lysates. Three independent experiments were performed, with similar results. (f) Ubiquitination levels upon transfection of the $\triangle 101-221-$ HA TRAP1 deletion mutant. HCT116 scramble, sh-TRAP1 and sh-TRAP1 cells transfected with the $\triangle 101-221$-HA TRAP1 mutant were sub-fractionated in PM (microsomes + CYTO fraction) and MITO fractions as described under Materials and Methods. Total lysates from the same cells were used as controls (left panel). Protein lysates were subjected to immunoblot analysis using mouse monoclonal anti-Ub antibodies to detect total ubiquitination levels. The purity of fractions was verified using mouse monoclonal anti-GAPDH (left and right panels) and mouse-monoclonal anti-COX IV (middle panel) antibodies. Three independent experiments were performed, with similar results. (g) Real-time RT-PCR analysis of BiP/Grp78 mRNA expression in scrambled and sh-TRAP1 HCT116 cells exposed to $1 \mu \mathrm{M}$ TG for $12 \mathrm{~h}$ (same as in Figure 2b) and in sh-TRAP1 HCT116 cells transfected with the $\Delta 1-59-M y c$ or $\triangle 101-221-H A$ TRAP1 mutant, as indicated, before treatment with TG. The $P$-values indicate the statistical significance between the BiP/Grp78 levels under the

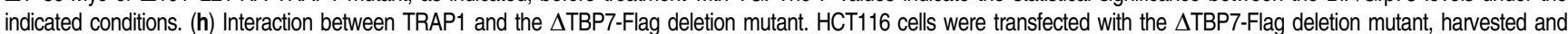
immunoprecipitated using anti-TRAP1 antibodies as described under Materials and Methods. Immunoprecipitates were separated by SDS-PAGE and immunoblotted using the indicated antibodies. No Ab, total cellular extracts incubated with $A / G$ plus agarose beads without antibody; IP, immunoprecipitation with the corresponding antibodies. Three independent experiments were performed, with similar results. The arrow indicates the $\Delta T B P 7-F l a g$ mutant band. (i) Ubiquitination levels upon transfection of the $\Delta T B P 7-F l a g$ deletion mutant. Total lysates from HCT116 scrambled cells transfected with $\triangle \mathrm{TBP7}$-Flag mutant were subjected to immunoblot analysis using mouse monoclonal anti-Ub antibodies to detect total ubiquitination levels and with mouse monoclonal anti-HSP60 antibodies for normalization of cell lysates. Three independent experiments were performed, with similar results 
a

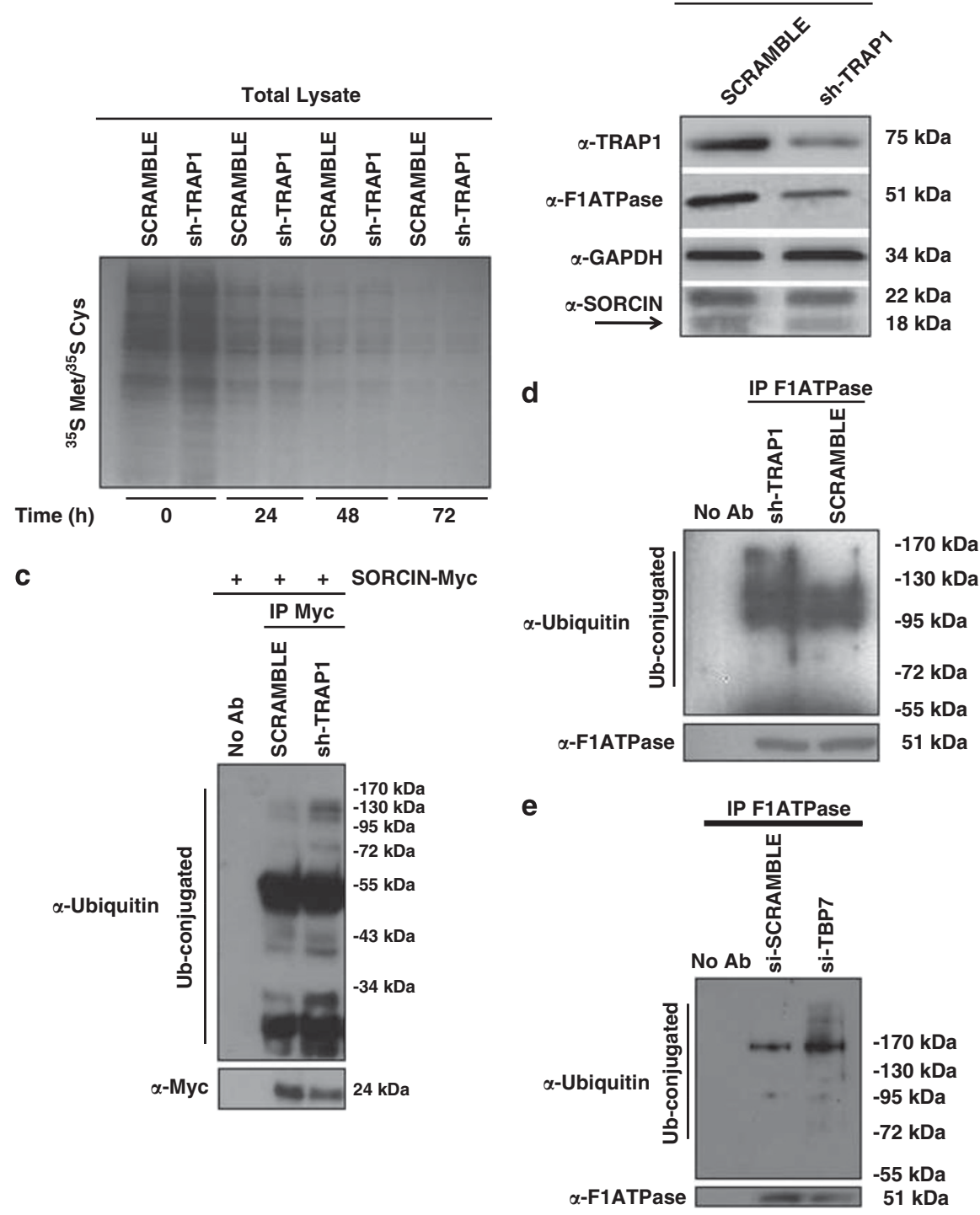

Figure 5 Control of intracellular protein stability and ubiquitination pattern of p18 Sorcin and F1ATPase. (a) Pulse-chase analysis of total lysates of scrambled and sh-TRAP1 HCT116 cells. HCT116 cells were incubated in cysteine/methionine-free medium for $1 \mathrm{~h}$ followed by incubation in cysteine/methionine-free medium containing $50 \mu \mathrm{Ci} / \mathrm{ml}^{35} \mathrm{~S}$-labeled cysteine/methionine $\left({ }^{35} \mathrm{~S}\right.$ Met $/{ }^{35} \mathrm{~S}$ Cys) for $1 \mathrm{~h}$. After labeling, cells were washed once with culture medium containing 10 -fold excess of unlabeled methionine and cysteine ( $5 \mathrm{mM}$ each) and incubated further in the same medium for the indicated time periods. Cells were collected at the indicated time points and total lysates subjected to SDS-PAGE were analyzed by autoradiography. (b) Total lysates of scrambled and sh-TRAP1 HCT116 cells were subjected to SDS-PAGE and immunoblotted using rabbit polyclonal anti-Sorcin, mouse monoclonal anti-TRAP1, goat polyclonal anti-F1ATPase antibodies. The same filter was re-probed using mouse monoclonal anti-GAPDH antibodies for normalization of cell lysates. The arrow indicates the MITO 18-kDa Sorcin isoform band. (c) Scrambled and sh-TRAP1 HCT116 clones were transfected with an expression vector containing the CDNA of p18 Sorcin fused to a c-Myc epitope at the C-terminus (Sorcin-Myc) and treated with $1 \mu \mathrm{M} \mathrm{MG132} \mathrm{for} 24 \mathrm{~h}$ before harvesting. Lysates were immunoprecipitated using mouse monoclonal anti-Myc antibodies and analyzed by immunoblot analysis using mouse monoclonal anti-Ub antibodies. The membrane was re-probed using an anti-Myc antibody to control transfection efficiency. (d) Scrambled and sh-TRAP1 HCT116 clones were treated with $1 \mu \mathrm{M}$ MG132 for $24 \mathrm{~h}$ before harvesting, immunoprecipitated with an-anti F1ATPase antibody, subjected to SDS-PAGE and immunoblotted using mouse monoclonal anti-Ub and goat polyclonal anti-F1ATPase antibodies. Three independent experiments were performed with similar results. (e) HCT116 cells were co-transfected with a Ub-HA vector and an siRNA negative control (scramble), or with siRNAs specific for TBP7; treated with $1 \mu \mathrm{M} \mathrm{MG132} \mathrm{for} 24 \mathrm{~h}$ before harvesting; immunoprecipitated using a goat polyclonal antiF1ATPase antibody; subjected to SDS-PAGE; and immunoblotted using mouse monoclonal anti-Ub and goat polyclonal anti-F1ATPase antibodies. Three independent experiments were performed, with similar results

mistargeted or misfolded, they are identified as aberrant and recognized by the Ub-proteasome system (UPS) for removal. This may even support a role of the CYTO UPS in controlling the levels and/or the quality of proteins destined for mitochondria. In addition, the presence of TRAP1 on the outer side of ER and the absence of TBP7 in mitochondria suggest that TRAP1 functions in these latter organelles are not directly linked to ubiquitination control, whereas this control is present in a different compartment and requires interaction with TBP7. In support of this model are results of transfection experiments using newly generated TRAP1 mutants, either able to interact with TBP7 and localized in the ER/cytosol fractions $(\Delta 1-59)$ or unable to bind to TBP7 but imported into mitochondria $(\Delta 101-221)$. Indeed, only the 


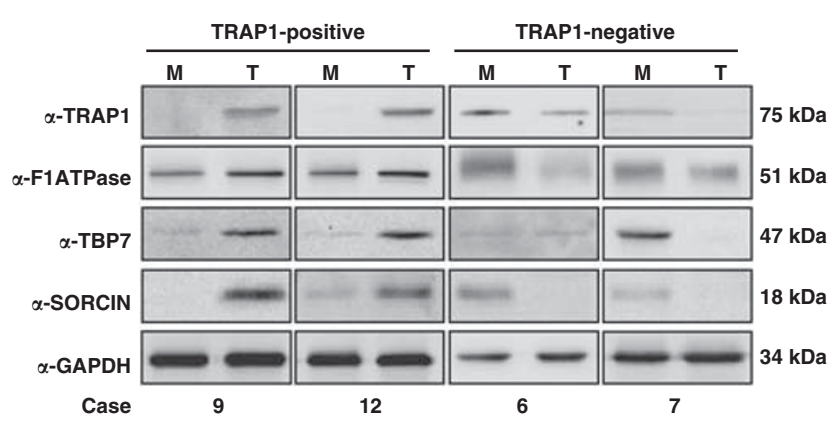

Figure 6 TRAP1, TBP7, F1ATPase and Sorcin expression in human CRCs. Total cell lysates from four human CRCs $(T)$ and the respective non-infiltrated peritumoral mucosas (M) were separated by SDS-PAGE and immunoblotted using rabbit polyclonal anti-Sorcin, mouse monoclonal anti-TRAP1, mouse monoclonal anti-TBP7 and goat polyclonal anti-F1ATPase. The same filter was re-probed using mouse monoclonal anti-GAPDH antibodies for normalization of cell lysates. For details on case numbers, refer to Supplementary Figure 1

MITO import-defective $\Delta 1-59$ mutant, and not the MITO $\Delta 101-221$ deletion mutant, rescues the strong Ub levels in sh-TRAP1-interfered cells. These findings provide proof of concept that protein quality control depends on the CYTO interaction between TRAP1 and TBP7. Consistent with these results is the observation that again only the $\triangle 1-59$ TRAP1 mutant rescues the decreased levels of TRAP1-regulated proteins (Supplementary Figure 3), as well as the levels of BiP/Grp78 mRNA upon TG-induced ER stress (Figure 4g). All these observations are in agreement with still unidentified proteasome members in mitochondria, even though several proteases, ATPases and Ub ligases have been identified. ${ }^{29}$ Furthermore, a functional interplay between MITO and proteasome activity has been demonstrated, thus suggesting that both systems are interdependent. ${ }^{30}$

Remarkably, the finding that the proposed TRAP1 network is conserved in CRCs is consistent with our model and provides new insights into the quality control/stability/ubiquitination of proteins in human cancer, a still highly debated issue. Indeed, the proteotoxic stress generated by accumulation of misfolded proteins and the consequent heat-shock response is currently under evaluation as a potential anticancer treatment target, as many tumor cells show constitutive proteotoxic stress and dependence on heat-shock response because of their rapid rates of proliferation and translation. ${ }^{31}$ Interestingly, bortezomib, a reversible inhibitor of the $26 \mathrm{~S}$ proteasome, is at present a valuable option for the first-line treatment of multiple myeloma. ${ }^{32}$ Thus, characterization of TRAP1, a chaperone upregulated in about $60 \%$ of human CRCs, ${ }^{5}$ as a protein involved in quality control and in protection against apoptosis in cancer cells provides a strong rationale for considering this network as a novel molecular target for treatment of human CRC.

In summary, a new crosstalk between ER and mitochondria is suggested and summarized in the working model, as shown in Figure 7. Our study demonstrates for the first time that TRAP1 is also present in the ER of cancer cells where it is involved in the quality control and intracellular protein ubiquitination of mitochondria-destined proteins, through direct interaction (as demonstrated by the FRET analysis shown in Figure 1d) with TBP7, one of the proteins present in the regulatory proteasome subunit. Thus, a 'customs office' could be hypothesized at the $E R /$ mitochondria interface, with TRAP1 and TBP7 being the officers at this important checkpoint. These two officers, each with independent but related functions, help to judge whether a protein can be repaired and reach its final MITO destination or, if the damage is too severe, it needs to be degraded.

\section{Materials and Methods}

Cell culture, plasmid generation and transfection procedures. HCT116 cells were cultured in DMEM supplemented with $10 \%$ fetal bovine serum (FBS) under standard conditions. Full-length TRAP1 and Sorcin expression vectors were obtained as described previously. ${ }^{12}$

Mutant $\triangle 1-59$-Myc was generated by using the following primers: $\triangle 1-59$-myc, forward: $5^{\prime}$-ATTAGAATTCATGAGCACGCAGACCGCCGAGG-3', reverse: $3^{\prime}-A T$ TACTCGAGGTGTCGCTCCAGGGCCTTGA-5'. PCR-amplified fragments were gel-purified and cloned in-frame into the pcDNA 3.1 plasmid (Invitrogen, San Giuliano Milanese, Italy) at the $E c o R I$ and Xhol restriction sites.

Mutant $\triangle 101-221-H A$ was generated by using the following primers: TRAP1HA, forward: 5 'attaGCGGCCGCGCAGCCAACATGGCGCGCGAGCCTGCGGG3', reverse: $5^{\prime}$-attaTCTAGATTAAGCGTAATCTGGAACATCATATGGGTATCAGT GTCGCTCCAGGGCCTTGA-3'; and $\triangle 101-221-H A$, forward: $5^{\prime}$-attaCCGCGGT CGGCAGCCCCGGGGAGCCT-3', reverse: $5^{\prime}$-attaCCGCGGAAACACCTCTTTTTC TGAGT $-3^{\prime}$. The PCR products obtained with the primers TRAP1-HA forward and $\triangle 101-221-H A$ reverse were cloned in the pRc-CMV vector (Invitrogen); the PCR product obtained with the primers $\triangle A T P a s e-H A$ forward and TRAP1-HA reverse was subcloned in the same plasmid. All clones were sequenced to confirm identity and PCR fidelity. The plasmid PCMV5L/S6 (TBP7-HA) was a gift from Dr Simon Dawson (University of Nottingham).

Mutant $\triangle \mathrm{TBP7}$-FLAG was generated by excising a fragment from the full-length TBP7 expression vector by using EcoRI and BamHI restriction endonucleases. The fragment was gel-purified and cloned into the corresponding sites of the expression vector p3x-FLAG.

Transient transfection of DNA plasmids was performed with the Polyfect Transfection reagent (Qiagen, Milan, Italy). siRNAs of TRAP1 and TBP7 were purchased from Qiagen (cat. no. S100301469 for TBP7 and cat. no. SI00115150 for TRAP1). For knockdown experiments, siRNAs were diluted to a final concentration of $20 \mathrm{nmol} / /$ and transfected according to the manufacturer's protocol. For control experiments, cells were transfected with a similar amount of scrambled siRNA (Qiagen; cat. no. SI03650318). Transient transfections of siRNAs were performed by using the HiPerFect Transfection Reagent (Qiagen). TRAP1-stable interference was achieved by transfecting HCT116 cells with TRAP1 (TGCTGTTGACAGT GAGCGACCCGGTCCCTGTACTCAGAAATAGTGAAGCCACAGATGTATTTCTG AGTACAGGGACCGGGCTGCCTACTGCCTCGGA) or scrambled (sequence containing no homology to known mammalian genes) shRNAs (Open Biosystems, Huntsville, AL, USA).

Cell extracts, purification and treatments. Total cell lysates were obtained by homogenization of cell pellets and tumor specimens in cold lysis buffer (20 mM Tris (pH 7.5), containing $300 \mathrm{mM}$ sucrose, $60 \mathrm{mM} \mathrm{KCl}, 15 \mathrm{mM} \mathrm{NaCl}, 5 \%$ (v) v) glycerol, $2 \mathrm{mM}$ EDTA, $1 \%$ (v/v) Triton X-100, $1 \mathrm{mM} \mathrm{PMSF}, 2 \mathrm{mg} / \mathrm{ml}$ aprotinin, $2 \mathrm{mg} / \mathrm{ml}$ leupeptin and $0.2 \%(\mathrm{w} / \mathrm{v})$ deoxycholate) for $1 \mathrm{~min}$ at $4{ }^{\circ} \mathrm{C}$ and further sonication for $30 \mathrm{~s}$ at $4{ }^{\circ} \mathrm{C}$. For ER stress induction, cells were treated overnight with $1 \mu \mathrm{M}$ TG (Sigma-Aldrich, Milan, Italy) before harvesting.

Mitochondria and ER were purified by using the Qproteome Mitochondria Isolation kit (Qiagen) according to the manufacturer's protocol and as described elsewhere. ${ }^{12}$ Briefly, HCT116 cells were washed and suspended in lysis buffer, which selectively disrupts the plasma membrane without solubilizing it, resulting in the isolation of CYTO proteins. Plasma membranes and compartmentalized organelles, such as nuclei, mitochondria and ER, remained intact and were pelleted by centrifugation. The resulting pellet was resuspended in disruption buffer, repeatedly passed through a narrow-gauge needle (to ensure complete cell disruption) and centrifuged to pellet nuclei, cell debris and unbroken cells. The supernatant (containing mitochondria and the microsomal fraction) was recentrifuged to pellet mitochondria. The resulting supernatant (microsomal fraction) was treated with proteinase-K for $20 \mathrm{~min}$ on ice \pm NP-40 (Igepal; Sigma-Aldrich) according to Hassink et al. ${ }^{33}$ or with $0.1 \mathrm{M} \mathrm{Na}_{2} \mathrm{CO}_{3}(\mathrm{pH} 11.3)$ for 30 min to remove peripheral ER membrane proteins. ${ }^{34}$ 


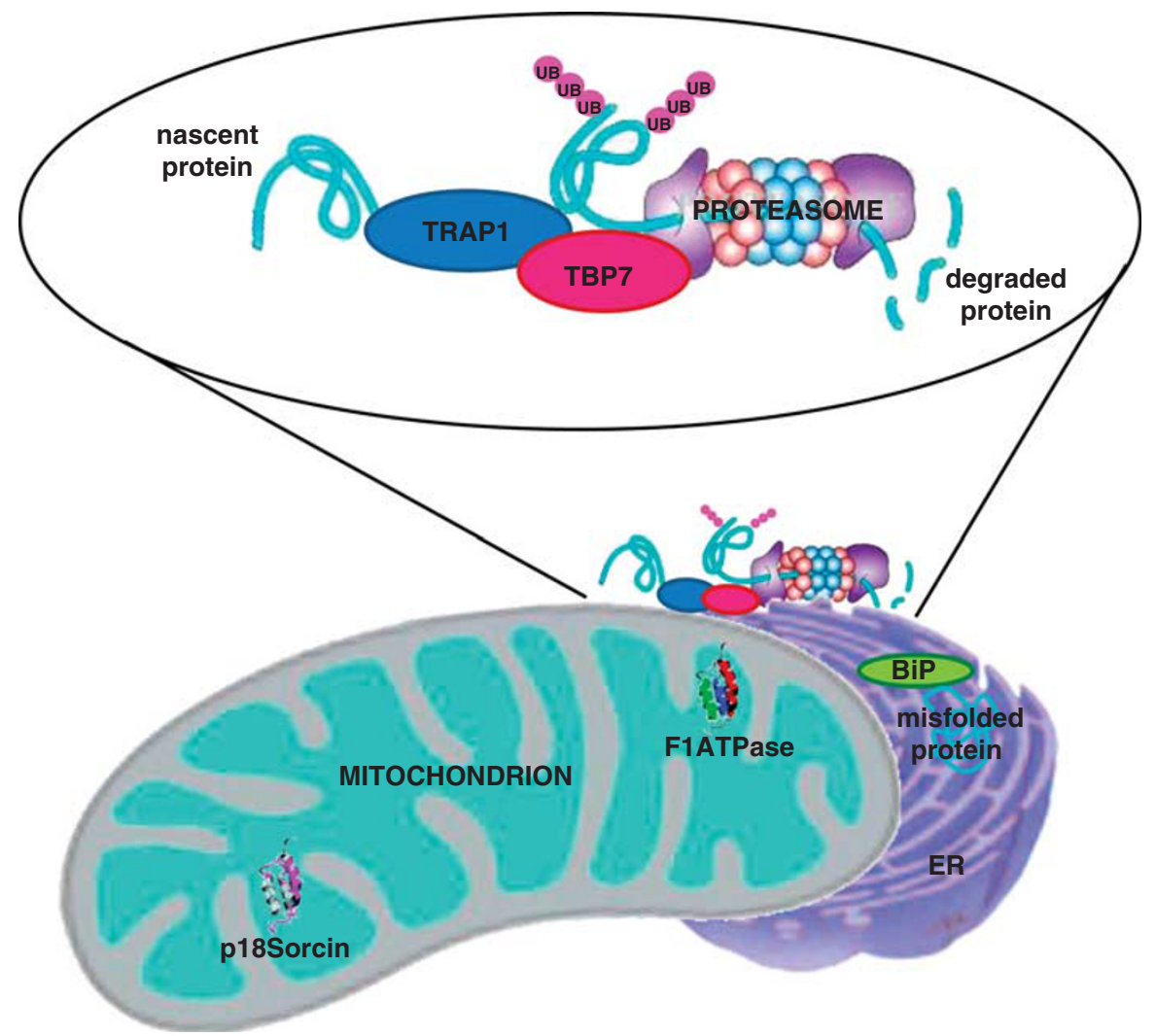

Figure 7 Crosstalk between ER and mitochondria, and MITO protein quality control. TRAP1 forms a supra-molecular complex with TBP7 on the outside of the ER, in a cellular compartment of tight ER-mitochondria contact sites, where proteasomes are also present. This TRAP1/TBP7 complex is involved in the control of protein stability and intracellular protein ubiquitination of mitochondria-destined proteins. These two proteins, each with independent but related functions, help to judge whether a protein can be repaired and reach the final MITO destination or, if damaged, needs to be degraded through the Ub-proteasome system

WB analysis and antibodies. Equal amounts of protein from cell lysates and tumor specimens were subjected to $10 \%$ (v/v) SDS-PAGE and transferred to a PVDF membrane (Millipore, Temecula, CA, USA). The membrane was blocked with $5 \%$ (w/v) skimmed milk and incubated with the primary antibody, followed by incubation with an HRP-conjugated secondary antibody. Proteins were visualized with an ECL detection system (GE Healthcare, Waukesha, WI, USA). The following antibodies from Santa Cruz Biotechnology (Segrate, Italy) were used for WB analysis and immunoprecipitation: anti-TRAP1 (sc-13557), anti-Sorcin (sc-100859), anti-TBP7 (sc-166003), anti-cMyc (sc-40), anti-CypD (sc-82570), anti-VDAC1 (sc-8830), anti-HSP60 (sc-1052), anti-Ub (sc-8017), anti-COX4 (sc-58348), anti-F1ATPase (ATP5B subunit; sc-58619), anti-tubulin (sc-8035), anti-HA (sc-805) and anti-glyceraldehyde-3-phosphate dehydrogenase (GAPDH; sc69778). A rabbit polyclonal anti-calnexin antibody (BD Biosciences, Milan, Italy) was also used.

RNA extraction and semi-quantitative and real-time RT-PCR analysis. Total RNA from cell pellets and tumor specimens was extracted by using the TRIzol Reagent (Invitrogen). For first-strand synthesis of cDNA, $3 \mu \mathrm{g}$ of RNA were used in a 20- $\mu$ l reaction mixture by using a cDNA Superscript II (Invitrogen). For real-time PCR analysis, $1 \mu \mathrm{l}$ of cDNA sample was amplified by using the Platinum SYBR Green qPCR Supermix UDG (Invitrogen) in an iCycler iQ Real-Time Detection System (Bio-Rad Laboratories $\mathrm{GmbH}$, Segrate, Italy). The following primers were used: BiP/Grp78, forward: $5^{\prime}$-CGTGGATGACCCGTCTGTG$3^{\prime}$, reverse: $5^{\prime}$-cTGCCGTAGGCTCGTTGATG-3' (PCR product $308 \mathrm{bp}$ ); and GA PDH, forward: 5'-CAAGGCTGAGAACGGGAA-3', reverse: 5'-GCATCGCCC CACTTGATTTT-3' (PCR product $90 \mathrm{bp}$ ). Primers were designed to be intronspanning. The reaction conditions were $50^{\circ} \mathrm{C}$ for $2 \mathrm{~min} ; 95^{\circ} \mathrm{C}$ for $2 \mathrm{~min}$; followed by 45 cycles of $15 \mathrm{~s}$ at $95^{\circ} \mathrm{C}, 30 \mathrm{~s}$ at $60^{\circ} \mathrm{C}$ and $30 \mathrm{~s}$ at $72^{\circ} \mathrm{C}$. GAPDH was chosen as an internal control.

For semi-quantitative RT-PCR, the RNA obtained by scrambled and sh-TRAP1 HCT116 cells was retro-transcribed and amplified using specific primers for $\mathrm{BiP} /$
Grp78 and GAPDH by using the Superscript III-One STEP kit (Invitrogen), according to the manufacturer's instructions. The following primers were used to amplify the corresponding transcripts: GAPDH, forward: $5^{\prime}$-GAAGGTGAAGGTCG GAGTC-3', reverse 5'-GAAGATGGTGATGGGATTTC-3'; and BiP/Grp78, forward: 5'-CTGGGTACATTTGATCTGACTGG-3', reverse: 5'-GCATCCTGGTGGCTTTCC AGCCATTC-3'. The primers for BiP/Grp78 were a gift from Professor P Remondelli (University of Salerno, Italy).

Apoptosis assay. HCT116 cells were subjected to downregulation of TRAP1 and TBP7 expression by siRNA transfection. Apoptosis was evaluated by cytofluorimetric analysis of Annexin-V and 7-amino-actinomycin-D (7-AAD)positive cells using the fluorescein isothiocyanate (FITC)-Annexin-V/7-AAD kit (Beckman Coulter, Milan, Italy). Stained cells were analyzed by using the 'EPICS XL' Flow Cytometer (Beckman Coulter). Ten thousand events were collected per sample. Positive staining for Annexin-V as well as double staining for Annexin-V and 7-AAD were interpreted as signs of, respectively, early and late phases of apoptosis. ${ }^{35}$ Experiments were performed three times using three replicates for each experimental condition.

Immunofluorescence, confocal microscopy and EM analysis. HCT116 cells were fixed with $0.1 \mathrm{M}$ phosphate buffer containing $4 \%(\mathrm{w} / \mathrm{v})$ paraformaldehyde for $15 \mathrm{~min}$, then blocked and permeabilized with $5 \%(\mathrm{w} / \mathrm{v}) \mathrm{BSA}$, $0.1 \%(\mathrm{v} / \mathrm{v})$ Triton X-100, 10\% (v/v) FBS in PBS for 20 min at RT before staining with primary antibodies (for TRAP1, CALNEXIN and TBP7) and the corresponding secondary TEXAS RED/FITC-conjugated antibodies. Immunofluorescence was analyzed by confocal laser-scanning microscopy using Zeiss 510 LSM (Carl Zeiss Microimaging, Göttingen, Germany), equipped with an Argon ionic laser (Carl Zeiss Microimaging) whose wavelength was set up to $488 \mathrm{~nm}$; a He-Ne laser whose wavelength was set up to $546 \mathrm{~nm}$; and an oil-immersion $\times 63 / 1.4 \mathrm{f}$ objective. For immuno-EM analysis, cells were fixed with a mixture of $4 \%(\mathrm{v} / \mathrm{v})$ paraformaldehyde and $0.05 \%(v / v)$ glutaraldehyde; labeled with a monoclonal antibody against HA by 
using the gold-enhance protocol; embedded in Epon-812; and cut as described previously. ${ }^{36} \mathrm{EM}$ images were acquired from thin sections by using an FEI Tecnai12 electron microscope equipped with an ULTRA VIEW CCD digital camera (FEI, Eindhoven, The Netherlands). Thin sections were also used for quantification of gold particles residing within mitochondria by using the AnalySIS software (Soft Imaging Systems GmbH, Munster, Germany).

FRET experiments. FRET was measured by using the acceptor photobleaching technique, ${ }^{20}$ where, upon irreversible photo-bleaching, the donor fluorescence increase was recorded. Cells on coverslips were fixed; immunostained with specific anti-TBP7 and anti-TRAP1 antibodies, and secondary antibodies conjugated, respectively, to Сy3 and Cy5; and mounted in PBS/glycerol (1:1). Images were collected using a laser-scanning confocal microscope (Zeiss LSM 510 Meta) equipped with a planapo $\times 63$ oil-immersion (NA 1.4) objective lens. Laser lines at 543 and $633 \mathrm{~nm}$ were used to excite, respectively, the fluorophores Cy3 and Cy5. For Cy5 bleaching, the 633-nm $\mathrm{He}-\mathrm{Ne}$ laser light with $100 \%$ output power was used and pinhole diameters were set to have $1.0-\mu \mathrm{m}$ optical slices.

FRET measurements were performed by using the LSM software (LSM Zeiss, Göttingen, Germany) after photo-bleaching of a selected squared ROI of $6 \mu \mathrm{m}^{2}$. We calculated the FRET efficiency on the basis of the following equation: $E=$ (Fluorescence intensity of Cy3 after bleaching-Fluorescence intensity of Cy3 before bleaching//Fluorescence intensity of Cy3 after bleaching. ${ }^{20}$

As control we measured FRET on cells expressing TBP7 alone labeled with Cy3 in order to ensure that photo-bleaching per se does not affect the fluorescence of the donor and that photo-conversion does not occur during the photo-bleaching analysis. We calculated the background raised by the photo-bleaching per se by bleaching Cy5 in cells negative for this fluorophore. The background value was subtracted from all samples.

Pulse-chase assay. Pulse-chase analysis was performed as described elsewhere. ${ }^{37}$ In brief, HCT116 cells were incubated in cysteine/methionine-free medium (Sigma-Aldrich) for $1 \mathrm{~h}$ followed by incubation in cysteine/methionine-free medium containing $50 \mu \mathrm{Ci} / \mathrm{ml}^{35} \mathrm{~S}$-labeled cysteine/methionine (GE Healthcare) for $1 \mathrm{~h}$. After labeling, cells were washed once with culture medium containing 10-fold excess of unlabeled methionine and cysteine ( $5 \mathrm{mM}$ each) and incubated further in the same medium for the indicated time periods. Cells were collected at the indicated time points and separated by $10 \%$ SDS-PAGE. Proteins were transferred onto a PVDF membrane (Millipore) and analyzed by autoradiography. The same filters were then probed by WB analysis.

Patients. Between May 2008 and May 2011, specimens from both tumor and normal, non-infiltrated peri-tumoral mucosa were obtained from 59 patients with CRC during surgical removal of the neoplasm. Samples were divided into $125-\mathrm{mm}^{3}$ pieces; one specimen was fixed in formalin and used for the histopathological diagnosis, whereas the others were immediately frozen in liquid nitrogen and stored at $-80^{\circ} \mathrm{C}$ for immunoblot analysis. Samples were analyzed within 30 days after collection and were thawed only once. Express written informed consent to use biological specimens for investigational procedures was obtained from all patients.

Statistical analysis. $\chi^{2}$-Test was used to establish statistical correlation between the expression levels of TRAP1 and those of Sorcin, F1ATPase and TBP7 in human CRCs. Statistically significant values $(P<0.05)$ are reported under section Results.

\section{Conflict of Interest}

The authors declare no conflict of interest.

Acknowledgements. This work was supported by the Associazione Italiana per la Ricerca sul Cancro (AIRC) (Grant IG8780), Ministero dell'Istruzione dell'Università e della Ricerca (PRIN 2008) and Fondazione Berlucchi to ML and FE. Our special thanks to Anthony Green for proofreading the manuscript and suggesting stylistic improvements, as well as to the Mass Spectrometry Unit (CEINGE Biotecnologie Avanzate, Naples, Italy), the Telethon Electron Microscopy Core Facility (TeEMCoF; IBP, CNR, Naples) and the Integrated Microscopy Facility (IGB, CNR, Naples) for EM assistance.
1. Song HY, Dunbar JD, Zhang YX, Guo D, Donner DB. Identification of a protein with homology to HSP90 that binds the type 1 tumor necrosis factor receptor. J Biol Chem 1995; 270: 3574-3581.

2. Felts SJ, Owen BA, Nguyen P, Trepel J, Donner DB, Toft DO. The HSP90-related protein TRAP1 is a mitochondrial protein with distinct functional properties. J Biol Chem 2000; 275 : 3305-3312

3. Montesano Gesualdi N, Chirico G, Catanese MT, Pirozzi G, Esposito F. AROS-29 is involved in adaptive response to oxidative stress. Free Radic Res 2006; 40: 467-476.

4. Montesano Gesualdi N, Chirico G, Pirozzi G, Costantino E, Landriscina M, Esposito F. Tumor necrosis factor-associated protein 1 (TRAP-1) protects cells from oxidative stress and apoptosis. Stress 2007; 10: 342-350.

5. Costantino E, Maddalena F, Calise S, Piscazzi A, Tirino V, Fersini A et al. TRAP1, a novel mitochondrial chaperone responsible for multi-drug resistance and protection from apoptosis in human colorectal carcinoma cells. Cancer Lett 2009; 279: 39-46.

6. Kang BH, Plescia J, Dohi T, Rosa J, Doxsey SJ, Altieri DC. Regulation of tumor cell mitochondrial homeostasis by an organelle-specific HSP9O chaperone network. Cell 2007; 131: 257-270.

7. Leav I, Plescia J, Goel HL, Li J, Jiang Z, Cohen RJ et al. Cytoprotective mitochondrial chaperone TRAP-1 as a novel molecular target in localized and metastatic prostate cancer. Am J Pathol 2010; 176: 393-401.

8. Kang BH, Siegelin MD, Plescia J, Raskett CM, Garlick DS, Dohi T et al. Preclinical characterization of mitochondria-targeted small molecule HSP90 inhibitors, gamitrinibs, in advanced prostate cancer. Clin Cancer Res 2010; 16: 4779-4788.

9. Kang BH, Tavecchio M, Goel HL, Hsieh CC, Garlick DS, Raskett CM et al. Targeted inhibition of mitochondrial HSP90 suppresses localised and metastatic prostate cancer growth in a genetic mouse model of disease. $\mathrm{Br} J$ Cancer 2011; 104: $629-634$.

10. Siegelin MD, Dohi T, Raskett CM, Orlowski GM, Powers CM, Gilbert CA et al. Exploiting the mitochondrial unfolded protein response for cancer therapy in mice and human cells. $J$ Clin Invest 2011; 121: 1349-1360.

11. Takemoto K, Miyata S, Takamura H, Katayama T, Tohyama M. Mitochondrial TRAP1 regulates the unfolded protein response in the endoplasmic reticulum. Neurochem Int 2011; 58: 880-887.

12. Landriscina M, Laudiero G, Maddalena F, Amoroso MR, Piscazzi A, Cozzolino F et al. Mitochondrial chaperone TRAP1 and the calcium binding protein Sorcin interact and protect cells against apoptosis induced by antiblastic agents. Cancer Res 2010; 70: 6577-6586.

13. Marx FP, Soehn AS, Berg D, Melle C, Schiesling C, Lang M et al. The proteasomal subunit S6 ATPase is a novel synphilin-1 interacting protein - implications for Parkinson's disease. FASEB J 2007; 21: 1759-1767

14. Kaneko T, Hamazaki J, lemura S, Sasaki K, Furuyama K, Natsume T et al. Assembly pathway of the mammalian proteasome base subcomplex is mediated by multiple specific chaperones. Cell 2009; 137: 914-925.

15. Landriscina M, Amoroso MR, Piscazzi A, Esposito F. Heat shock proteins, cell survival and drug resistance: the mitochondrial chaperone TRAP1, a potential novel target for ovarian cancer therapy. Gynecol Oncol 2010; 117: 177-182.

16. Landriscina M, Maddalena F, Laudiero G. Esposito F. Adaptation to oxidative stress, chemoresistance, and cell survival. Antioxid Redox Signal 2009; 11: 2701-2716.

17. Truax AD, Koues OI, Mentel MK, Greer SF. The 19S ATPase S6a (S6'/TBP1) regulates the transcription initiation of class II transactivator. J Mol Biol 2010; 395: 254-269.

18. Tsai YC, Weissman AM. The unfolded protein response, degradation from endoplasmic reticulum and cancer. Genes Cancer 2010; 1: 764-778.

19. Ohana B, Moore PA, Ruben SM, Southgate CD, Green MR, Rosen CA. The type 1 human immunodeficiency virus Tat binding protein is a transcriptional activator belonging to an additional family of evolutionarily conserved genes. Proc Natl Acad Sci USA 1993; 90: $138-142$

20. Kenworthy AK, Edidin M. Imaging fluorescence resonance energy transfer as probe of membrane organization and molecular associations of GPI-anchored proteins. Methods Mol Biol 1999; 116: 37-49.

21. Enyedi B, Várnai $P$, Geiszt $M$. Redox state of the endoplasmic reticulum is controlled by Ero1L-alpha and intraluminal calcium. Antioxid Redox Signal 2010; 13: 721-729.

22. Masuda Y, Shima G, Aiuchi T, Horie M, Hori K, Nakajo $S$ et al. Involvement of tumor necrosis factor receptor-associated protein 1 (TRAP1) in apoptosis induced by betahydroxyisovalerylshikonin. J Biol Chem 2004; 279: 42503-42515.

23. Morales AP, Carvalho AC, Monteforte PT, Hirata H, Han SW, Hsu YT et al. Endoplasmic reticulum calcium release engages Bax translocation in cortical astrocytes. Neurochem Res 2011; 36: 829-838.

24. Chen WT, Lee AS. Measurement and modification of the expression level of the chaperone protein and signaling regulator GRP78/BiP in mammalian cells. Methods Enzymol 2011; 490: 217-233.

25. Hayashi T, Su TP. Sigma-1 receptor chaperones at the ER-mitochondrion interface regulate $\mathrm{Ca}(2+)$ signaling and cell survival. Cell 2007; 131: 596-610.

26. Azzu V, Brand MD. Degradation of an intramitochondrial protein by the cytosolic proteasome. J Cell Sci 2010; 123 (Part 4): 578-585.

27. Baker BM, Haynes $\mathrm{CM}$. Mitochondrial protein quality control during biogenesis and aging. Trends Biochem Sci 2011; 36: 254-261. 
28. Livnat-Levanon N, Glickman MH. Ubiquitin-proteasome system and mitochondria reciprocity. Biochim Biophys Acta 2011; 1809: 80-87.

29. Germain D. Ubiquitin-dependent and -independent mitochondrial protein quality controls: implications in ageing and neurodegenerative diseases. Mol Microbiol 2008; 70: $1334-1341$

30. Kozieł R, Greussing R, Maier AB, Declercq L, Jansen-Dürr P. Functional interplay between mitochondrial and proteasome activity in skin aging. J Invest Dermatol 2011; 131: 594-603.

31. Neznanov N, Komarov AP, Neznanova L, Stanhope-Baker P, Gudkov A Proteotoxic stress targeted therapy (PSTT): induction of protein misfolding enhances the antitumor effect of the proteasome inhibitor bortezomib. Oncotarget 2011; 2 : 209-221.

32. Ludwig H, Beksac M, Bladé J, Cavenagh J, Cavo M, Delforge M et al. Multiple myeloma treatment strategies with novel agents in 2011: a European perspective. Oncologist 2011; 16: $388-403$.
33. Hassink GC, Zhao B, Sompallae R, Altum M, Gastaldello S, Zinin NV et al. The ER-resident ubiquitin-specific protease19 participates in the UPR and rescues ERAD substrates. EMBO Rep 2009; 10: 755-761.

34. Fujiki Y, Hubbard AL, Fowler S, Lazarow PB. Isolation of intracellular membranes by means of sodium carbonate treatment: application to endoplasmic reticulum. J Cell Biol 1982; 93: 97-102.

35. George TC, Basiji DA, Hall BE, Lynch DH, Ortyn WE, Perry DJ et al. Distinguishing modes of cell death using the ImageStream multispectral imaging flow cytometer. Cytometry $A$ 2004; 59: 237-245

36. Polishchuk EV, Di Pentima A, Luini A, Polishchuk RS. Mechanism of constitutive export from the Golgi: bulk flow via the formation, protrusion, and en bloc cleavage of large transGolgi network tubular domains. Mol Biol Cell 2003; 14: 4470-4485.

37. Lieberman AP, Harmison G, Strand AD, Olson JM, Fischbeck KH. Altered transcriptional regulation in cells expressing the expanded polyglutamine androgen receptor. Hum $\mathrm{Mol}$ Genet 2002; 11: 1967-1976.

Supplementary Information accompanies the paper on Cell Death and Differentiation website (http://www.nature.com/cdd) 